\title{
Self-Organized Percolation Power Laws with and without Fractal Geometry in the Etching of Random Solids
}

\author{
Agnès Desolneux $^{\dagger}{ }^{1}$, Bernard Sapoval ${ }^{\Uparrow}{ }^{1,2}$, and Andrea Baldassarri ${ }^{\S} 1-3$
}

\begin{abstract}
Classically, percolation critical exponents are linked to the power laws that characterize percolation cluster fractal properties. It is found here that the gradient percolation power laws are conserved even for extreme gradient values for which the frontier of the infinite cluster is no more fractal.

In particular the exponent $7 / 4$ which was recently demonstrated to be the exact value for the dimension of the so-called "hull" or external perimeter of the incipient percolation cluster, controls the width and length of gradient percolation frontiers whatever the gradient magnitude.

This behavior is extended to previous model studies of etching by a finite volume of etching solution in contact with a disordered solid. In such a model, the dynamics stop spontaneously on an equilibrium self-similar surface similar to the fractal frontier of gradient percolation. It is shown that the power laws describing the system geometry involves also the fractal dimension of the percolation hull, whatever the value of the dynamically generated gradient, i.e. even for a non-fractal frontier.

The comparison between numerical results and the exact results that can be obtained analytically for extreme values of the gradient suggests that there exist a unique power law valid from the smallest possible scale up to infinity. These results suggest the possible existence of an underlying conservation law, relating the length and the statistical width of percolation gradient frontiers.
\end{abstract}

\section{Introduction: Gradient percolation built By Diffusion or By ETCHING}

Spreading of objects in space with a gradient of occupation probability is most common. From chemical composition gradients to the distribution of plants which depend on their solar exposure, probability gradients exist in many inhomogeneous systems. In fact inhomogeneity is a rule in nature whereas most of the systems that physicists are studying are homogeneous as they are thought to be more simple to understand. In particular phase transitions or critical phenomena are studied in that framework, the simplest being percolation transition [1. In this work, we study a different situation, that of an inhomogeneous system, the gradient percolation situation.

1991 Mathematics Subject Classification. Primary 62B43; Secondary 82B20, 60K37. 


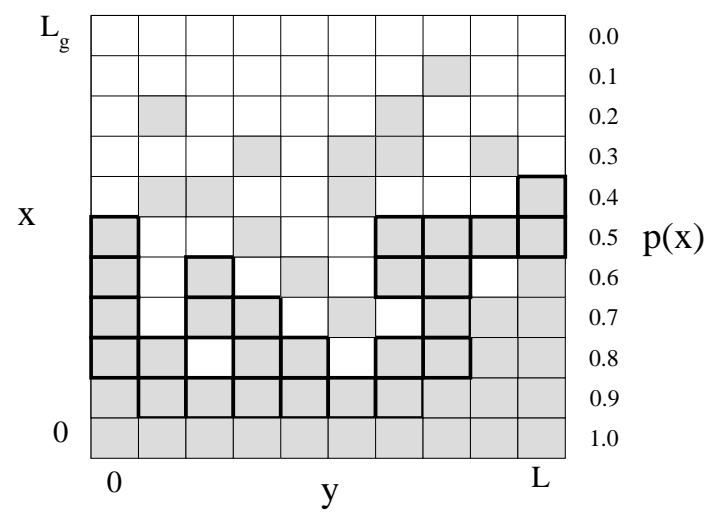

Figure 1. The Gradient Percolation Model. Each row has an occupation probability $p(x)$ ranging from 1 at the bottom to 0 on the top. A site with coordinates $(x, y)$ is occupied with probability $p(x)$. The occupied and empty sites are represented respectively in grey and white. Apart from isolated islands and lakes, grey and white sites form two distinct connected regions. The marked sites (with thick borders) are the connected front of the occupied percolating cluster.

First we study a paradigmatic situation of such an inhomogeneous system, the Gradient Percolation GP model 2, 3. We show how the scaling behavior, which usually characterizes criticality, extends down to minimal possible scales, i.e. the extreme gradient regime. In this regime, exact analytical analysis of the model is possible and given here.

Moreover this analysis can be extended to other models, as the etching gradient percolation dynamical model EGP, which has recently been shown to belong to the same universality class as GP. In this case, the extreme gradient analysis allows to correctly recognize such a universality (and its limitations) without the explicit knowledge of the underlying dynamically generated gradient.

\section{Gradient Percolation GP.}

GP Definition of Gradient Percolation. The gradient percolation (GP) situation was first introduced in the study of diffusion fronts which exists for instance as the consequence of a soldering process [2, 3. It is defined in Fig. 1 and examples are shown in Fig. 2 The figures give examples of a random distribution of points on a lattice with a linear gradient of concentration in the vertical direction.

It is a $2 \mathrm{D}$ square lattice of size $L_{g} \times L$, where each point $(x, y)$ is occupied with probability $p(x)=1-x / L_{g}$ ( $x$ being the vertical direction in the figure). In gradient percolation there is always an infinite cluster of occupied sites as there is a region where $p$ is larger than the standard percolation $\mathbf{S P}$ threshold $p_{c}$. There is also an infinite cluster of empty sites as there is a region where $p$ is smaller than $p_{c}$. The object of interest is the GP front, the external limit (or frontier) of the infinite occupied cluster. 

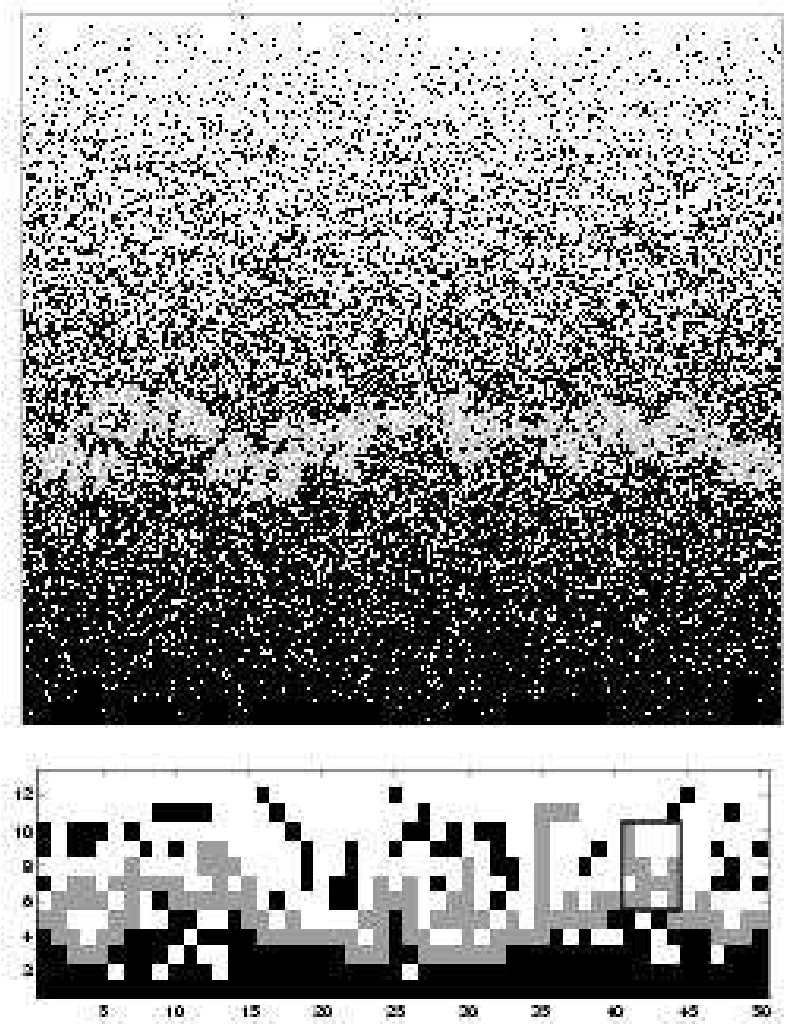

Figure 2. Gradient percolation (GP) front. Particles are distributed at random with probability $p(x)=1-x / L_{g}$. The occupied sites are in black and the last line of connected occupied sites is the gradient percolation front shown in light gray. This situation corresponds also to a diffusion situation where the front is called the diffusion front. Top: $L=L_{g}=500$. Bottom: $L=50$ and $L_{g}=12$. The black window has an horizontal width equal to $\sigma_{f}$. It contains approximately $L_{g}$ points.

Its precise definition depends on the lattice geometry:

- Triangular Lattice. The GP front is the connected set of sites belonging to the occupied infinite cluster which are first nearest neighbours to sites of the infinite empty cluster.

- Square Lattice. The GP front is the connected set of sites belonging to the occupied infinite cluster which are first or second nearest neighbours with sites of the infinite empty cluster, itself defined through a first and second neighbours connection.

The square lattice case is shown in grey in Fig. 2 This front is a random object with an average position $x_{f}$, a statistical width $\sigma_{f}$ and a total length $N_{f}$. In so far that the GP front and the SP external perimeter (often called hull) have the same geometry their fractal dimension was first conjectured to be exactly equal to $7 / 4$ 


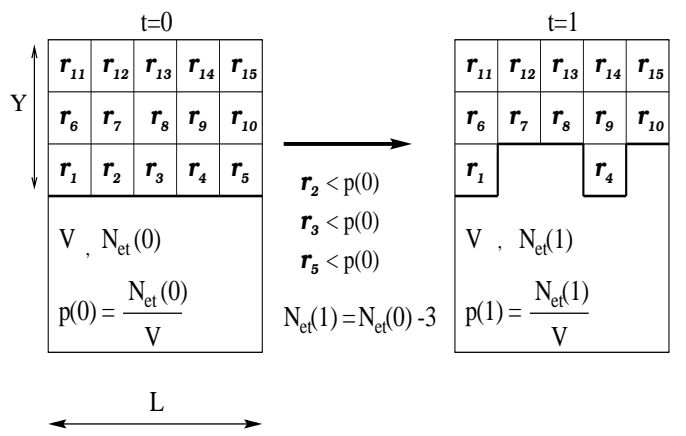

FiguRE 3. Sketch of the etching dynamics in a square lattice: sites $2,3,5$ are etched at the first time-step as their resistances are smaller than $p(0)$. Consequently the number of etchant particles in the solution decreases by 3 units. At $t=1$, the new interface sites than can possibly be etched are $7,8,10$ if the solution can etch only the first nearest neighbours. If the solution can also etch second nearest neighbours in a diagonal direction the whole second layer can possibly be etched.

in 2. This result was then demonstrated heuristically by Saleur and Duplantier [4] and very recently it was proved mathematically by Smirnov and Werner [5].

Etching Gradient Percolation EGP. The same type of fractal geometry has been recently found in a very different physical situation. It first appeared in experiments [6]. It was interpreted in a model in which an etching solution is in contact with the initially flat surface of a disordered solid and starts to corrode its weakest regions. The solid surface then gets "harder" but at the same time new regions are discovered which contain weak elements. Often the corrosive power of the solution is proportional to an etchant concentration and if the etchant is consumed in the reaction, the corrosive power of a finite volume of solution decreases during the time evolution of the process. As the solid surface gets harder and harder, and the corroding power gets weaker and weaker, the corrosion process stops spontaneously in a finite time interval.

The EGP model definition. We first recall the two-dimensional etching model introduced in 7. Its schematic is shown in Fig. 3

- A 2D random solid is represented as a site lattice (triangular or square), of linear width $L$ and, eventually, infinite depth.

- A random number $r_{i} \in[0,1]$ (extracted from the flat probability density function $\pi_{0}(r)=1$ for $\left.r \in[0,1]\right)$ is assigned to each solid site $i$, representing its resistance to the etching by the solution. $r_{i}$ does not depend on time (quenched disorder), or on the site environment.

- The etching solution has a volume $V$ and is initially in contact with the solid through the bottom boundary (see Fig. 3). It contains an initial number $N_{e t}(0)$ of dissolved etchant molecules.

Consequently, the initial concentration $C(0)$ of etchant in the solution is given by: $C(0)=N_{e t}(0) / V$. Calling $N_{e t}(t)$ the number of etchant molecules at time $t$, 


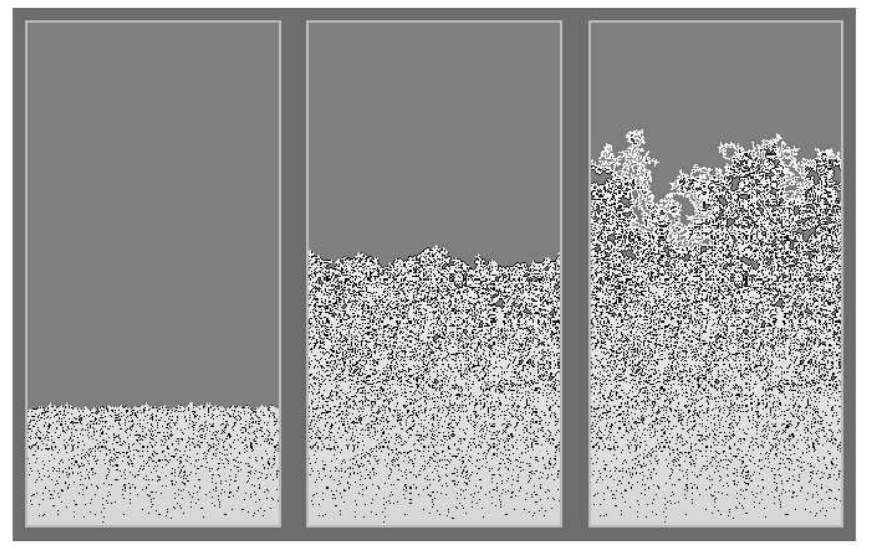

FiguRE 4. Etching process for two intermediate times and final equilibrium situation. The solid is shown in grey, the solution in white, and the finite size solid clusters that are detached by the etching in black. The final solid surface is found to be fractal with dimension $7 / 4$ up to a characteristic scale $\sigma_{f}$.

$C(t)=N_{e t}(t) / V$. At each time-step, the "etching power" of the solution (i.e. the average "force" exerted by the solution on a solid surface particle) is supposed to be proportional to $C(t): p(t)=\Gamma C(t)$. Hereafter the assumption $\Gamma=1$ is made, without loss of generality. It implies $C(t) \equiv p(t)$. At time-step $t$, all the interface sites with $r_{i}<p(t)$ are dissolved and a particle of etchant is consumed for each of these corroded solid sites.

The etching evolution is depicted in Fig. 4. A brief review of the known results, will be given in the next section.

The structure of this paper is the following: in the next section we recall definitions and known results for fractal "weak gradient" cases. Then we consider the extreme gradient case for GP. In that case a few exact results are given. They are discussed in the spirit of deciding whether or not there exists power laws for GP that are valid from the smallest scale to infinity.

They are then compared with numerical results obtained for small size systems. Next the EGP is studied numerically for large gradients. The results are then extrapolated to large (fractal) systems and a remarkable agreement is found between extrapolated values and numerical values. Gradient percolation results are then compared with etching gradient percolation. In the conclusion we discuss to what extent the numerical results give evidence that there exists a new conservation law in diffusion or gradient percolation, this law being valid from the smallest scale to infinity. 


\section{GP And EGP Known Results}

2.1. Gradient Percolation (GP): Known Results. The early GP studies were focussed at finding its relations with standard percolation. Let us first recall the definitions. For $0 \leq x \leq L_{g}, n_{f}(x)$ is the mean number of points of the front lying on the line $x$ per unit horizontal length. It measures the front density at distance $x$. The length $N_{f}$, the position $x_{f}$ and the width $\sigma_{f}$ of the front are then defined in terms of $n_{f}(x)$ by

$$
\begin{gathered}
N_{f}=L \sum_{x=0}^{L_{g}} n_{f}(x), \quad x_{f}=\frac{\sum_{x=0}^{L_{g}} x n_{f}(x)}{\sum_{x=0}^{L_{g}} n_{f}(x)}, \\
\text { and } \sigma_{f}^{2}=\frac{\sum_{x=0}^{L_{g}}\left(x-x_{f}\right)^{2} n_{f}(x)}{\sum_{x=0}^{L_{g}} n_{f}(x)} .
\end{gathered}
$$

It was found that the mean front was located at a distance where the density of occupation was very close to $p_{c}$ or $p\left(x_{f}\right) \simeq p_{c}$. This was verified numerically with such precision that the gradient percolation method is now often used to compute percolation thresholds $[\mathbf{8}, \mathbf{9}, \mathbf{1 0}, \mathbf{1 1}, \mathbf{1 2}$. It was also found that:

(1) The width $\sigma_{f}$ depends on $L_{g}$ through a power law $\sigma_{f} \propto\left(L_{g}\right)^{\nu /(1+\nu)}$ where $\nu=4 / 3$ is the correlation length exponent [1] in dimension $d=2$ so that $\sigma_{f}=\left(L_{g}\right)^{4 / 7}$. The width $\sigma_{f}$ was also shown to be a percolation correlation length.

(2) Secondly it was found that the front was fractal with a dimension $D_{f}$, numerically determined, close to 1.75. The front length followed a power law $N_{f} \propto\left(L_{g}\right)^{\alpha_{N}}$ with $\alpha_{N}=\left(D_{f}-1\right) \nu /(1+\nu)$.

(3) But also, it was numerically observed that the sum of these two exponents was very close to 1 . If true, this meant that $\nu /(1+\nu)+\left(D_{f}-1\right) \nu /(1+\nu)=1$ or $D_{f}=1+1 / \nu=7 / 4$. This is how it was conjectured in [2] that $D_{f}=7 / 4$.

In that sense the ordinary GP power laws were thought to be linked to the SP exponent $\nu$ and to the fractality of the percolation cluster hull. Up to now, these facts were considered to be strictly valid only in the large system limit.

However, if true, and we know now that $7 / 4$ is the exact value, there follows an intriguing relation, namely $\sigma_{f}^{D_{f}}$ is exactly proportional to $L_{g}$. This means that the number of surface particles within the correlation length is exactly proportional to $L_{g}$. This is particularly striking for diffusion fronts. Diffusion of particles from a source results in a concentration gradient and an associated GP situation. In that frame, the above result means that, if $L_{g}$ particles have diffused on a vertical row, there is on average the same (or a constant fraction of) number of particles on the correlated surface (surface content of a box with a lateral size equal to the statistical width). This fact seems a priori to have nothing to do with scaling, percolation and the thermodynamic limit. From this point of view it is possibly the consequence of a conservation law and if such a conservation exists, it should apply also for extreme gradients corresponding to $L_{g}$ of a few units.

In particular it should apply to the very extreme $L_{g}=1,2$ and 3 for which exact values of $x_{f}, N_{f}$ and $\sigma_{f}$ can be calculated analytically. The corresponding results are the subject of section 3 . 


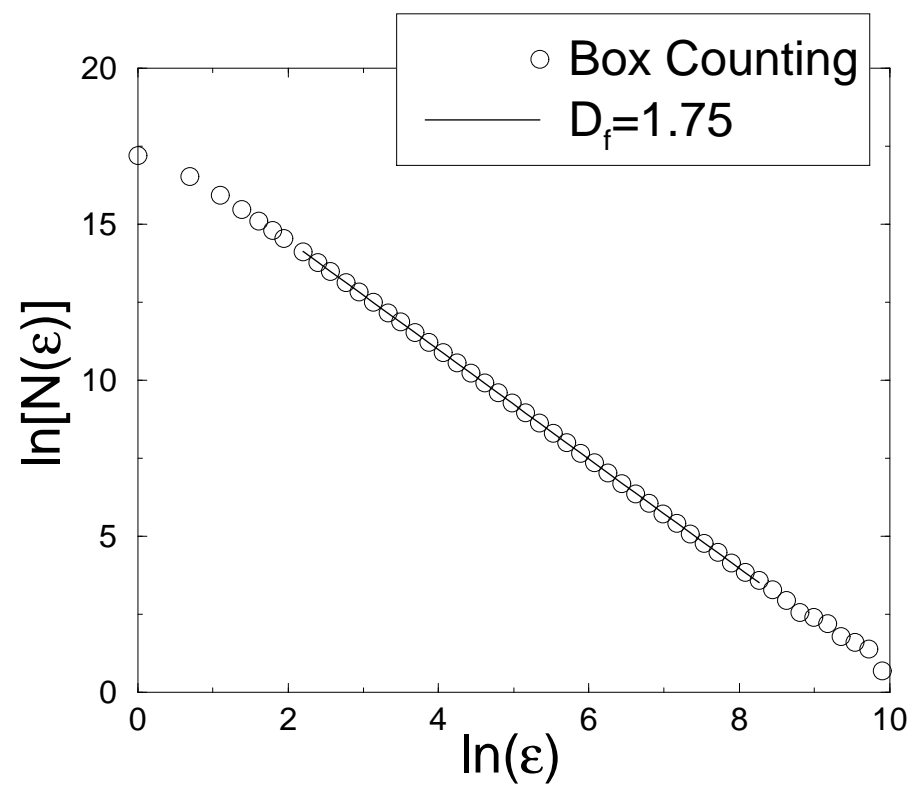

Figure 5. Box-counting determination of the fractal dimension $D_{f}$ of the corrosion front ( $\epsilon$ is the linear size of the box, $N(\epsilon)$ the number of boxes containing at least one point of the front). The value of $D_{f}=1.753 \pm 0.005$ is found fit for values of $\epsilon$ ranging from a few lattice distances to the front width $\sigma_{f}$ (in this case $\sigma_{f} \approx 3000$, i.e. $\left.\ln \left(\sigma_{f}\right) \approx 8\right)$.

2.2. Etching Gradient Percolation (EGP): Known Results. Let us call $n(t)$ the number of dissolved solid sites at time-step $t$. One can express several quantities through $n(t)$, or its time-integral $N(t)$, that is the total number of corroded solid sites up to time $t$. The number of etchant particles in the liquid will decrease as:

$$
N_{e t}(t+1)=N_{e t}(t)-n(t)=N_{e t}(0)-N(t),
$$

and consequently the etching power of the solution is:

$$
p(t+1)=p(t)-\frac{n(t)}{V}=p(0)-\frac{N(t)}{V} .
$$

Note that, as $p(t+1)<p(t)$, a site having resisted to etching at a certain time-step will resist forever. Consequently, the part of the solid surface which can be etched at time-step $t+1$ is restricted to the sites which have been just uncovered by the etching process at time $t$. We call this subset of surface the "active" part of the surface. After a given time-step, all the solid sites which have been previously explored by the solution are "passive" forever. However it may happen that "passive" sites are disconnected from the bulk at a later time-step if they are connected to the solid by weak sites.

The model reproduces qualitatively the same phenomenology observed experimentally [6]. The dynamical evolution can be divided into two different regimes: 


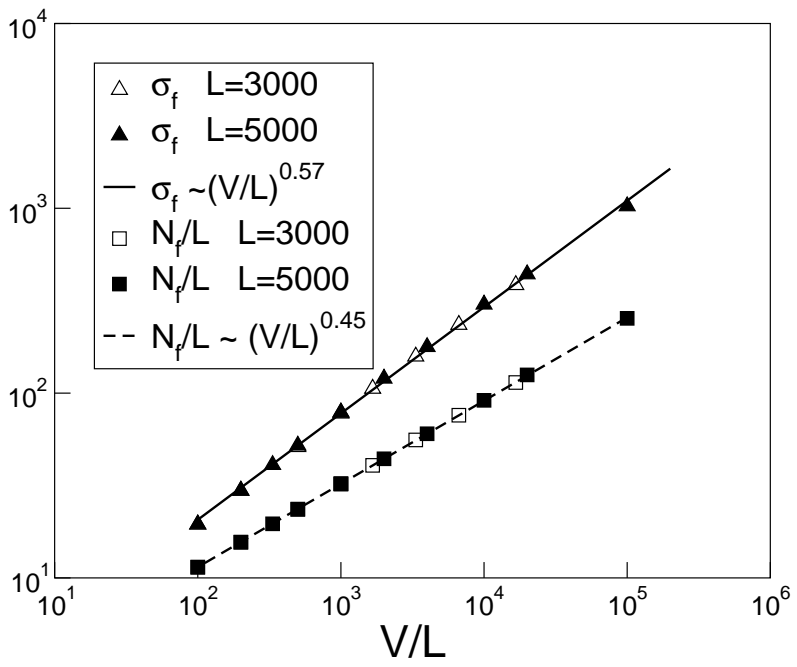

Figure 6. Behavior of $\sigma_{f}$ and of the average number of sites per column $N_{f} / L$ as a function of $V / L$ for several sample sizes (several values of $L$ ).

(1) In the first (smooth) regime, the corrosion is well directed while the front becomes progressively rougher and rougher. In our model this regime does not depend on the details of the discretization, or on the fundamental geometrical features of the lattice, like the embedding space dimension or the lattice coordination number.

(2) In the second regime, the correlations revealed by the hardening process become important: the dynamics becomes locally isotropic, generating a fractal front. This corresponds to a critical regime, directly related to the percolation transition on the same lattice.

In the case of etching the same results as for GP were found on the final front geometry. The fractal dimension $D_{f}$ of the etching front was measured (up to the scale $\sigma_{f}$ ) using the box-counting [13] algorithm. In this way $D_{f}=1.753 \pm 0.005$ is measured (see Fig. 囵) very close to the value $7 / 4$ of GP.

In fact it was shown 14 that in EGP the length $V / L$, ratio of the solution volume by the sample width, plays the role of $L_{g}$. The width and length of the front were found to follow the same power laws as in GP, as shown in Fig. [6 In particular $\sigma_{f}$ scales as $(V / L)^{\alpha_{\sigma}}$, with $\alpha_{\sigma} \approx 0.57$, while $N_{f} / L$ scales as $(V / L)^{\alpha_{N}}$, with an observed value for $\alpha_{N} \approx 0.45$.

The scaling relation $\alpha_{\sigma}+\alpha_{N}=1$ seems also fairly obeyed, but a direct inspection give an even better result, as shown in Fig. [7 where $\sigma_{f} N_{f} / L$ is plotted as a function of $V / L$. A power law fit gives an exponent very close to one, and correspondingly, a linear fit gives equivalently good results.

As the same relations are verified by EGP, there should also exist a conservation law for that case. This law would stipulate that the amount of correlated final front is proportional to the ratio of the solution volume divided by the lateral size of the sample, in other words to the depth of the solution. Formulated in this manner 


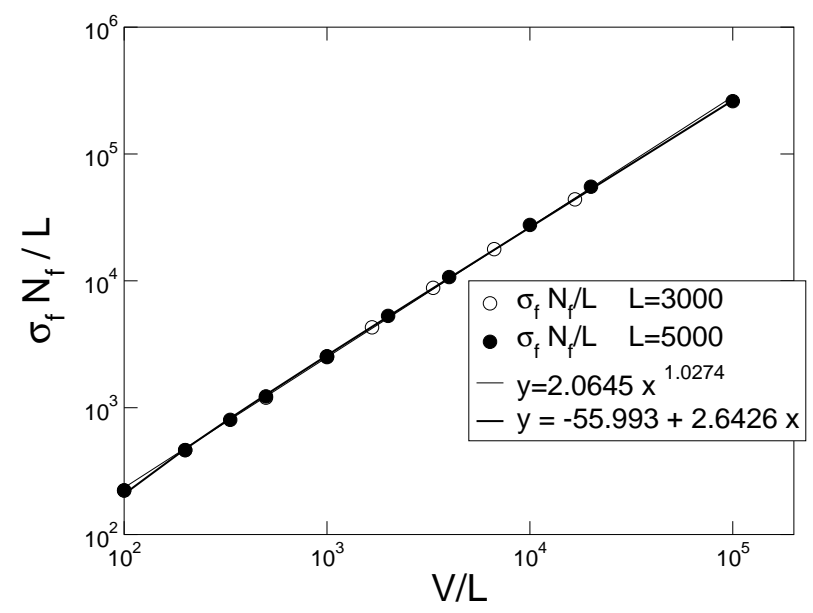

Figure 7. Behavior of $\sigma_{f} N_{f} / L$ as a function of $V / L$. Data are fitted with a power law, resulting in an exponent very close to one, and with a linear law, resulting in comparable results.

there is no reason why this property should not be verified for very small solution volumes, as shown in the next section.

\section{Extreme Gradients: fractal exponents Without fractal GEOMETRY}

In this section we will show how, even in the case of extreme gradient (hence in absence of any fractal structure) one recovers the above power laws.

3.1. Exact calculations for extreme GP. We present a detailed description of some exact results obtained for $L_{g}=2,3$, hence the two highest non trivial value for the gradients.

3.1.1. Triangular lattice.

Exact computations for $L_{g}=2$. We find occupied sites only on the lines $x=0$ and $x=1$. For $1 \leq i \leq L$, let $X_{i}$ be the random variable which has value 1 if the $\mathrm{i}^{t h}$ site of the line $x=1$ is occupied, and 0 otherwise. Then, the $X_{i}$ 's are independent, and each takes value 1 with probability $1 / 2$ (see Fig. [8).

- Since all the occupied sites on the line $x=1$ belong to the front, we have $N_{f}(1)=\sum_{i=1}^{L} X_{i}$. The mean number of points of the front on this line is thus $\mathbf{E}\left(N_{f}(1)\right)=L / 2$.

- On the line $x=0$ all the sites are occupied, but a site will belong to the front if and only if at least one of its two neighbours on the line $x=1$ is not occupied (see Fig. 8). Thus, $N_{f}(0)=L-\sum_{i=1}^{L} X_{i} X_{i+1}$, and the mean number of points of the front on the line $x=0$ is then $\mathbf{E}\left(N_{f}(0)\right)=L-L / 4=3 L / 4$.

Finally, we get

$$
n_{f}(0)=\frac{3}{4} \text { and } n_{f}(1)=\frac{1}{2}
$$




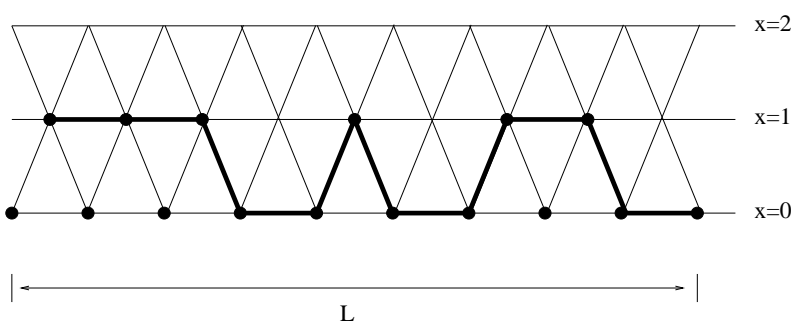

FiguRE 8. Gradient percolation on the triangular lattice with $L_{g}=2$ (the sites on the line $x=1$ are occupied with probability $1 / 2$ ). Thick disks represent occupied sites, and the thick line joins the sites belonging to the GP front.

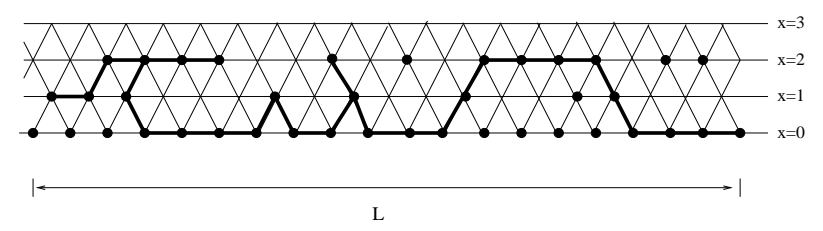

Figure 9. Gradient percolation on the triangular lattice with $L_{g}=3$ (the sites on the line $x=1$ are occupied with probability $2 / 3$, and the ones on the line $x=2$ with probability $1 / 3$ ). Thick disks represent occupied sites, and the thick line joins the sites belonging to the front.

and thus we can compute the mean length $N_{f}$, the mean position $x_{f}$ and the width $\sigma_{f}$ of the front, and find:

$$
\begin{aligned}
& \frac{N_{f}}{L}=\frac{5}{4}=1.25, \quad \sigma_{f}=\frac{\sqrt{6}}{5}=0.4898979 \ldots, \\
& x_{f}=\frac{2}{5}=0.4 \text { and } p\left(x_{f}\right)=1-\frac{x_{f}}{L_{g}}=0.8 .
\end{aligned}
$$

Exact computations for $L_{g}=3$. For $1 \leq i \leq L$, let $X_{i}$ be the random variable which has value 1 if the $\mathrm{i}^{\text {th }}$ site of the line $x=2$ is occupied, and 0 otherwise. Then, the $X_{i}$ 's are independent, and each takes value 1 with probability $1 / 3$. In the same way, we also define $Y_{i}$ as the random variable which has value 1 if the $\mathrm{i}^{\text {th }}$ site of the line $x=1$ is occupied, and 0 otherwise. The $Y_{i}$ 's are also independent, and each takes value 1 with probability $2 / 3$ (see Fig. 9).

- A site on the line $x=2$ will belong to the front if it is occupied and if it does not belong to an isolated cluster (i.e. a cluster of occupied sites on the line $x=2$, surrounded by empty sites, see Fig. 9). We can write this in terms of $X_{i}$ and $Y_{i}$ in the following way: $N_{f}(2)=X_{1}+\ldots+X_{L}-$ $\sum_{i=1}^{L} \sum_{n \geq 1} n\left(1-X_{i-1}\right) X_{i} \ldots X_{i+n-1}\left(1-X_{i+n+1}\right)\left(1-Y_{i}\right) \ldots\left(1-Y_{i+n}\right)$. The mean number of points of the front lying on the line $x=2$ is thus

$$
\mathbf{E}\left(N_{f}(2)\right)=\frac{1}{3} L-L \times \frac{4}{9} \times \frac{1}{3} \times \sum_{n \geq 1} n \frac{1}{9^{n}}=\frac{5}{16} L .
$$


- A site on the line $x=1$ will belong to the front if it is occupied and if: either at least one of its two neighbours on the line $x=2$ is empty, either these two neighbours are occupied but the site is connected to the "sea" on its left or on its right (see for example, on Fig. 9 the third occupied site from the left on the line $x=1$ ). Thus,

$$
N_{f}(1)=\sum_{i=1}^{L} Y_{i}\left(1-X_{i} X_{i-1}\right)+\sum_{i=1}^{L} Y_{i} X_{i} X_{i-1}\left(S_{i}+S_{i}^{\prime}-S_{i} S_{i}^{\prime}\right),
$$

where $S_{i}=\sum_{n \geq 1}\left(1-Y_{i-1}\right) \ldots\left(1-Y_{i-n}\right) X_{i-2} \ldots X_{i-n}\left(1-X_{i-n-1}\right)$ is a binary ( 0 or 1 ) random variable which has value 1 when the site $i$ (on the line $x=1$ ) is connected on its left to the "sea". $S_{i}^{\prime}$ is the analogous of $S_{i}$ for the connection on its right. We notice that $S_{i}^{\prime}$ and $S_{i}$ are independent, identically distributed, and their mean value is $\mathbf{E}\left(S_{i}\right)=\mathbf{E}\left(S_{i}^{\prime}\right)=$ $\sum_{n \geq 1}(1 / 3)^{n}(1 / 3)^{n-1} \times 2 / 3=1 / 4$.

The mean number of points of the front lying on the line $x=1$ is thus

$$
\mathbf{E}\left(N_{f}(1)\right)=\frac{2}{3}\left(1-\frac{1}{9}\right) L+\frac{2}{3} \times \frac{1}{9}\left(2 \times \frac{1}{4}-\frac{1}{4^{2}}\right) L=\frac{5}{8} L .
$$

- A site on the line $x=0$ will not belong to the front if: else, its two neighbours on the line $x=1$ are occupied, or else, one of these two neighbours is empty but the site is not connected to the "sea" (this is for example the case of three consecutive sites of the line $x=0$ on the right of Fig. 9). Thus, we have: $N_{f}(0)=L-\sum_{i=1}^{L} Y_{i} Y_{i+1}-\sum_{i=1}^{L} \sum_{n \geq 1}(n+$ 1) $Y_{i}\left(1-Y_{i+1}\right) \ldots\left(1-Y_{i+n}\right) Y_{i+n+1} X_{i} X_{i+1} \ldots X_{i+n}$. Then, the mean number of points of the front lying on the line $x=0$ is

$$
\mathbf{E}\left(N_{f}(0)\right)=L-\frac{4}{9} L-L \times \frac{4}{9} \times \frac{1}{3} \sum_{n \geq 1}(n+1) \frac{1}{9^{n}}=\frac{223}{432} L .
$$

To summarize, we get

$$
n_{f}(0)=\frac{223}{432}, \quad n_{f}(1)=\frac{5}{8} \text { and } n_{f}(2)=\frac{5}{16},
$$

and then we can compute the mean length $N_{f}$, the mean position $x_{f}$ and the width $\sigma_{f}$ of the front, and find:

$$
\begin{gathered}
\frac{N_{f}}{L}=\frac{157}{108}=1.453704 \ldots, \sigma_{f}=\frac{9 \sqrt{335}}{157 \sqrt{2}}=0.7419084 \ldots, \\
x_{f}=\frac{135}{157}=0.8598726 \ldots \text { and } p\left(x_{f}\right)=0.7133758 \ldots .
\end{gathered}
$$

3.1.2. Square lattice. The situation for the square lattice is a little different from the situation for the triangular lattice, because of the problem of the choice between 4-connexity and 8-connexity. In our case, the definition of the front will be the following: the front is the set of occupied sites which are connected through occupied 4-neighbours to the line $x=0$, and have an empty 8-neighbours which belongs to the empty 8 -connected component of the line $x=L_{g}$. 

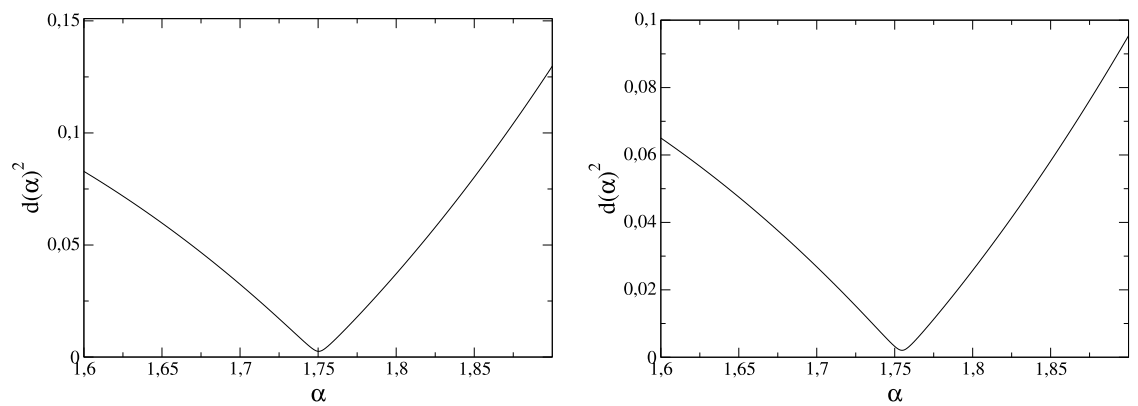

Figure 10. Determination of the best exponent value: Left, square lattice: $\alpha=1.750$; Right: triangular lattice, $\alpha=1.754$.

Exact computations for $L_{g}=2$. The computations for $L_{g}=2$ in the case of the square lattice are very similar to the ones in the triangular case. All the occupied sites of the line $x=1$ belong to the front, and a site on the line $x=0$ belongs to the front if at least one of is three neighbours on the line $x=1$ is empty. Thus, we get

$$
n_{f}(0)=1-\frac{1}{2^{3}}=\frac{7}{8} \text { and } n_{f}(1)=\frac{1}{2},
$$

and then we can compute the mean length $N_{f}$, the mean position $x_{f}$ and the width $\sigma_{f}$ of the front:

$$
\begin{gathered}
\frac{N_{f}}{L}=\frac{11}{8}=1.375, \sigma_{f}=\frac{2 \sqrt{7}}{11}=0.4810457 \ldots \\
x_{f}=\frac{4}{11}=0.36363636 \ldots \text { and } p\left(x_{f}\right)=0.81818181 \ldots . .
\end{gathered}
$$

Exact computations for $L_{g}=3$. For $L_{g}=3$, the computations are similar to the ones in the case of the triangular lattice, but a little more complicated since a site has more neighbours, and thus more different geometric configurations have to be considered. But it can be done, and we finally get:

$$
n_{f}(0)=\frac{8029}{11664}, \quad n_{f}(1)=\frac{47}{72} \text { and } n_{f}(2)=\frac{13}{48},
$$

and for the mean length $N_{f}$, the mean position $x_{f}$ and the width $\sigma_{f}$ of the front, we find:

$$
\begin{gathered}
\frac{N_{f}}{L}=\frac{9401}{5832}=1.611968 \ldots, \sigma_{f}=\frac{9 \sqrt{576049}}{9401}=0.7266046 \ldots \\
x_{f}=\frac{6966}{9401}=0.7409850 \ldots \text { and } p\left(x_{f}\right)=0.7530050 \ldots .
\end{gathered}
$$

3.2. Numerical results. The problem is then to compare the numerical GP laws to these exact values. As will be shown, the numerical results verify the above power laws with such precision that the question arises of the existence of a simple mathematical power law extending from $L_{g}=1$ to infinity. To try to answer this question we proceed in two steps. First we test these laws on the numerical results obtained for $L_{g}$ between 4 and 50 for the square and triangular lattices by searching the best numerical power laws followed by the width. Considering arbitrary exponents $\alpha$ between 1.6 and 1.9, we study $\sigma_{f}^{\alpha}$ as a function of $L_{g}$ between 

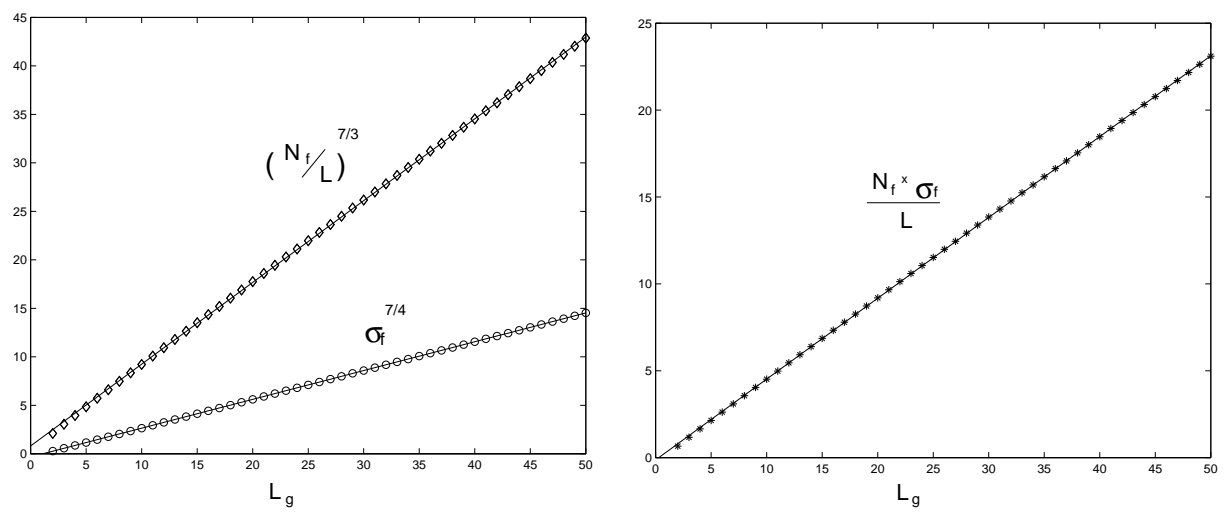

Figure 11. Numerical results for the square lattice. Left: the circles resp. diamonds represent respectively $\sigma_{f}^{7 / 4}$ and $\left(N_{f} / L\right)^{7 / 3}$. Right: the stars represent $N_{f} \times \sigma_{f} / L$. The lines are the ones obtained by linear fit on the data for $L_{g}=4$ to 50 .

\begin{tabular}{|c|c|c|c|}
\hline square lattice & $L_{g}=1$ & $L_{g}=2$ & $L_{g}=3$ \\
\hline exact $\sigma_{f}$ & 0 & 0.4810 & 0.7266 \\
\hline$\sigma_{f}(4-50)$ & -0.13 & 0.47 & 0.72 \\
\hline$\sigma_{f}(4-5)$ & -0.106 & 0.478 & 0.726 \\
\hline$\delta \sigma_{f}(4-5)$ & 0.025 & 0.006 & 0.002 \\
\hline exact $N_{f} / L$ & 1 & 1.3750 & 1.6120 \\
\hline$N_{f} / L(4-50)$ & 1.24 & 1.48 & 1.68 \\
\hline$N_{f} / L(4-5)$ & 1.109 & 1.393 & 1.615 \\
\hline$\delta\left(N_{f} / L\right)(4-5)$ & 0.017 & 0.009 & 0.004 \\
\hline
\end{tabular}

TABLE 1. Comparison between exact and extrapolated results for the square lattice. The data $(4-50)$ resp. $(4-5)$ correspond to extrapolated values from the respective ranges $(4-50)$ resp. $(4-5)$ (see text). $\delta$ is the confidence interval.

4 and 50. For each $\alpha$ value, there is a best line $\sigma_{f}^{\alpha}=a_{\alpha}\left(L_{g}+b_{\alpha}\right)$ fitting the numerical $\sigma_{f}^{\alpha}$. The introduction of the term $b_{\alpha}$ is justified by the fact that when a power law is verified for large systems it includes always the possibility that a small (as compared to the system size) term could contribute but in a negligible manner. But here the size itself is small or very small. On the other hand, one should remark that for $L_{g}=1$ the width is strictly 0 so that some negative value of $b_{\alpha}$ should be present. In the next step, the mean error $d(\alpha)$, defined by $d(\alpha)^{2}=$ $(1 / 47) \sum_{L_{g}=4}^{50}\left(\sigma_{f}\left(L_{g}\right)^{\alpha}-a_{\alpha}\left(L_{g}+b_{\alpha}\right)\right)^{2}$, is measured numerically as a function of $\alpha$. The results are shown in Fig. 10] There is a clear minimum for $\alpha \approx 1.75$, showing that this exponent gives the best power law fit. Once the best fit with the empirical data is made one has the best values for the parameters $a$ and $b: a=0.297$ and $b=-1.094$. Note that $b$ should be strictly equal to -1 in order to obtain a null width for the trivial case $L_{g}=1$. 


\begin{tabular}{|c|c|c|c|}
\hline triangular lattice & $L_{g}=1$ & $L_{g}=2$ & $L_{g}=3$ \\
\hline exact $\sigma_{f}$ & 0 & 0.4899 & 0.7419 \\
\hline$\sigma_{f}(4-50)$ & -0.15 & 0.48 & 0.74 \\
\hline$\sigma_{f}(4-5)$ & -0.151 & 0.480 & 0.740 \\
\hline$\delta \sigma_{f}(4-5)$ & 0.019 & 0.006 & 0.002 \\
\hline exact $N_{f} / L$ & 1 & 1.2500 & 1.4537 \\
\hline$N_{f} / L(4-50)$ & 1.16 & 1.36 & 1.46 \\
\hline$N_{f} / L(4-5)$ & 1.014 & 1.262 & 1.457 \\
\hline$\delta\left(N_{f} / L\right)(4-5)$ & 0.015 & 0.008 & 0.004 \\
\hline
\end{tabular}

TABLE 2. Comparison between exact and extrapolated results for the triangular lattice. The data $(4-50)$ resp. $(4-5)$ correspond to extrapolated values from the respective ranges $(4-50)$ resp. $(4-5)$ (see text). $\delta$ is the confidence interval.

Another verification of the extreme GP power laws can be obtained from the study of the front length or of the quantity $\left(N_{f} / L\right)^{7 / 3}$ as a function of $L_{g}$. In Fig. 11] (left plot), the diamonds represent the values of $\left(N_{f} / L\right)^{7 / 3}$ and the best linear fit has equation $Y=c\left(L_{g}+d\right)$ with $c=0.843$ and $d=0.959$. This shows indeed that the exponents $4 / 7$ and $3 / 7$ can be used down to the steepest gradients for which the frontier is no more fractal.

We also study the quantity $N_{f} \times \sigma_{f} / L$ as a function of $L_{g}$ : the result is shown on Fig. 11 (right plot). The best linear fit (on the values from $L_{g}=4$ to 50 has equation $Y=\tilde{c}\left(L_{g}+\tilde{d}\right)$ with $\tilde{c}=0.465$ and $\tilde{d}=-0.278$.

One can then extrapolate the $\sigma_{f}$ values to the case $L_{g}=1,2$ and 3 . The results are given in Table 1 for both lattices. One observes that the numerical extrapolations correspond to the exact values with an apparent good precision. However no firm conclusion can be drawn without discussion of the numerical uncertainties. The values shown on Fig. [11] are averaged over 100 trials on a length $L=5.10^{5}$. As the fit occurs through a power law it is difficult to give a confidence interval for the coefficients $a$ and $b$ (obtained from a least square linear regression on the values of $\left.\sigma_{f}^{7 / 4}\right)$.

In order to obtain a better control on the numerical precision of $a$ and $b$ we made extensive computations of the two cases $L_{g}=4$ and $L_{g}=5$ with 100 trials on a length $L=5.10^{5}$. Doing so we obtain the mean values with their standard deviation: $\sigma_{f}(4)^{7 / 4}=0.8658 \pm 0.0009$ and for $\sigma_{f}(5)^{7 / 4}=1.1610 \pm 0.0013$. Thus if we compute the equation of the line $\bar{a}\left(L_{g}+\bar{b}\right)$ which interpolates the two points $\left(4, \sigma_{f}(4)^{7 / 4}\right)$ and $\left(5, \sigma_{f}(5)^{7 / 4}\right)$, we obtain $\bar{a}=0.2952 \pm 0.0022$ and $\bar{b}=-1.066 \pm 0.041$. This last result shows that the value -1 is compatible with $\bar{b}$ and its statistical error. Given the numerical values for $L_{g}=4$ and 5 , we can also get extrapolated values for $\sigma_{f}$ for $L_{g}$ smaller, together with their confidence interval. The result (see Tables 1 and 21) is that the predicted values are very close to the exact ones. For $\left(N_{f} / L\right)^{7 / 3}$, in the same way we obtain a linear interpolation of the values for $L_{g}=4$ and 5 , with coefficients $\bar{c}=0,893 \pm 0.014$ and $\bar{d}=0.427 \pm 0.074$.

A comparison of the main fitting results for the two geometries (square and triangular) is shown in Table 3 . 


\begin{tabular}{|c|c|c|}
\hline numerical results & $\begin{array}{c}\text { square lattice } \\
100 \times 500000\end{array}$ & $\begin{array}{c}\text { triangular lattice } \\
100 \times 500000\end{array}$ \\
\hline$\sigma_{f}^{7 / 4}$ fit for & $a=0.297$, & $a=0.315$, \\
data $L g=4 \rightarrow 50$ & $b=-1.094$ & $b=-1.113$ \\
\hline$\left(N_{f} / L\right)^{7 / 3}$ fit for & $c=0.843$, & $c=0.636$, \\
data $L g=4 \rightarrow 50$ & $d=0.959$ & $d=1.214$ \\
\hline$\sigma_{f} \times N_{f} / L$ fit for & $\tilde{c}=0.465$, & $\tilde{c}=0.426$, \\
data $L g=4 \rightarrow 50$ & $\tilde{d}=-0.278$ & $\tilde{d}=-0.198$ \\
\hline exact linear fit from & $a=0.2940$, & $a=0.3062$, \\
$\sigma_{f}^{7 / 4}(2)$ and $\sigma_{f}^{7 / 4}(3)$ & $b=-1.0548$ & $b=-1.0632$ \\
\hline
\end{tabular}

TABLE 3. Numerical results of the linear fit for the square and triangular lattices. The data are obtained from the averaged over 100 trials with a length $L=500000$.

3.3. Extreme Gradients for EGP. To check the extreme gradient regime for EGP numerical simulations have been performed for large systems $(L \geq 5000)$ in a wide range of etchant volumes $V$. As discussed in [14], the ratio $L / V$ is proportional to the self-established gradient generated by the dynamical process, at least in the small gradient regime. Here we show how it is possible to check the whole range of gradients (from small to extreme) without explicitly knowing the exact value of the gradient.

Let us assume that there exists such a parameter $g$, or equivalently $L_{g}=1 / g$, such that $\sigma_{f} \propto L_{g}^{4 / 7}$ and $N_{f} \propto L_{g}^{3 / 7}$. In such a case, one should have, along the lines discussed above:

$$
\sigma_{f}^{7 / 4}=A\left(\frac{N_{f}}{L}\right)^{7 / 3}+B
$$

In order to check the validity of the above relation on the entire gradient range, we proceeding in the following manner. First, we use relation (3) to determine the parameter $A$ and $B$ fitting the data on an extreme gradient (non fractal) range. Once the parameters $A$ and $B$ have been determined, we use, we use relation 3 to check the agreement with the numerical results obtained for small gradients, in the fractal regime. In Fig. 12 the outcome of such a procedure is shown, in the case of a triangular lattice. In the first graph (top left) the fitting region is shown. The data correspond to the simulation of more than 100 realizations of systems sized $L \geq 10000$ for $V$ ranging from $V=L$ to $V \approx 3 L$. The resulting values of $N_{f} / L$ correspond, in the GP model, to $L_{g}$ ranging approximatively from 1 to 70 . The result of the fit is $A=0.58804$ and $B=-0.89278$. The following four graphs (from left to right in the first and then in the second rows) display the values predicted by the linear relation (3) (straight line) compared with the simulation results (for a system sized $L=5000$ ). Each graph corresponds to a scale ten times larger than the previous graph. Finally, the last graph (bottom right) shows the whole range of simulation results together with the linear relation (3), in a log-log scale.

3.4. Comparison between Gradient Percolation and Etching Gradient Percolation. The process of creation of the interface being different, the question arises of the comparison between the two geometries. For this we compare the 

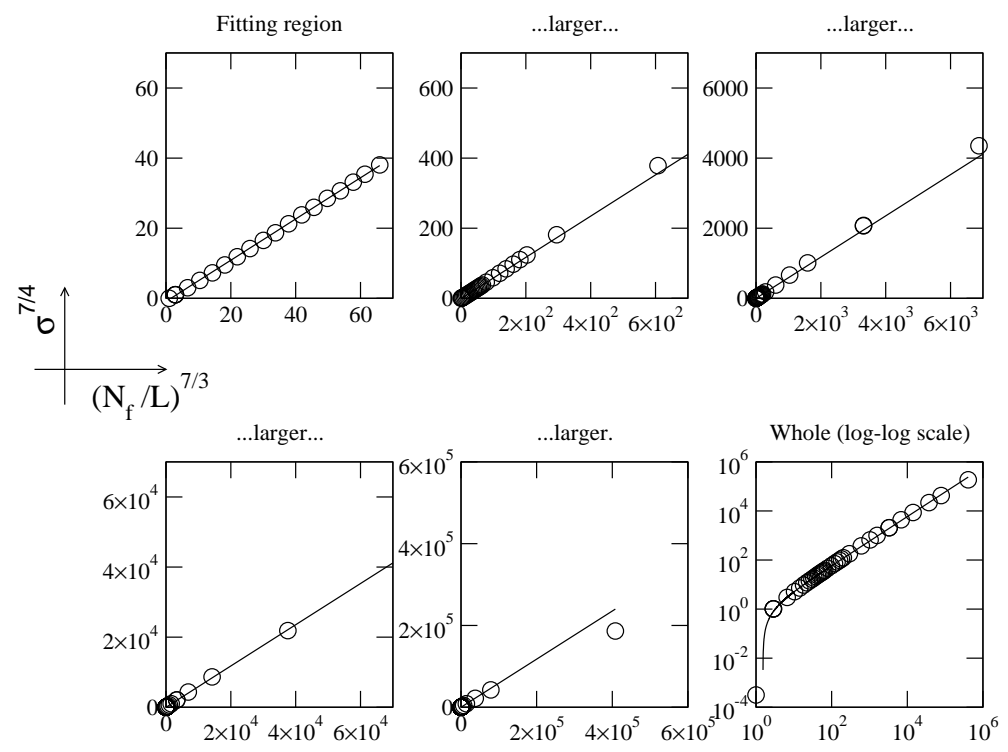

FiguRE 12. E.G.P numerical scaling extrapolated from the extreme gradient regime. In the first graph (top-left) the data are fitted against a linear relation. Then the predicted values for larger ranges are compared with simulations. The last graph (bottomright) displays the whole range of data, in a log-log plot.

extreme gradient results of the two models. In Fig. 13 we show the numerical results for GP and EGP for both lattices. The result of the linear fit, with the relation (3) are displayed in Table 4

First note that, as expected, the slopes are different for the two lattices. More interestingly, given a lattice geometry, the GP model and the EGP model have slightly different coefficients $A$ and $B$. This shows that GP and EGP are not strictly equivalent, even if they belong to the same universality class (with respect to exponents). The differences between GP and EGP can be explained in the following way. In EGP the probability of invasion by the solution is applied on the previous irregular frontier while in GP the probability changes row by row. In that sense in EGP the front penetration may be slightly increased as compared to GP.

Another quantity that displays a difference between the two models, is the depth (i.e. x-coordinate) distribution of the front sites, as shown in Fig. 14] Note that, at odds with the GP case, the EGP is asymmetric. To understand such an asymmetry one has to recall the mechanism that creates a gradient in EGP. There, at each time $t$ there is an etching power $p(t)$ and the front is at an average distance $x(t)$. This means that $p$ depends implictly on $x$, through the dummy variable $t$. Consequently, there is an effective "self-generated" gradient, but there is no reason why this gradient should be constant as it is the case for standard GP. In particular in the last period of etching, $p$ varies only weakly below $p_{c}$ [14 while the penetration still increases. This means that the gradient corresponding to the last period is smaller, inducing a wider distribution for the front. Alternatively if GP was studied with a concave $p(x)$ (decreasing gradient) the frontier would naturally be asymmetric. 


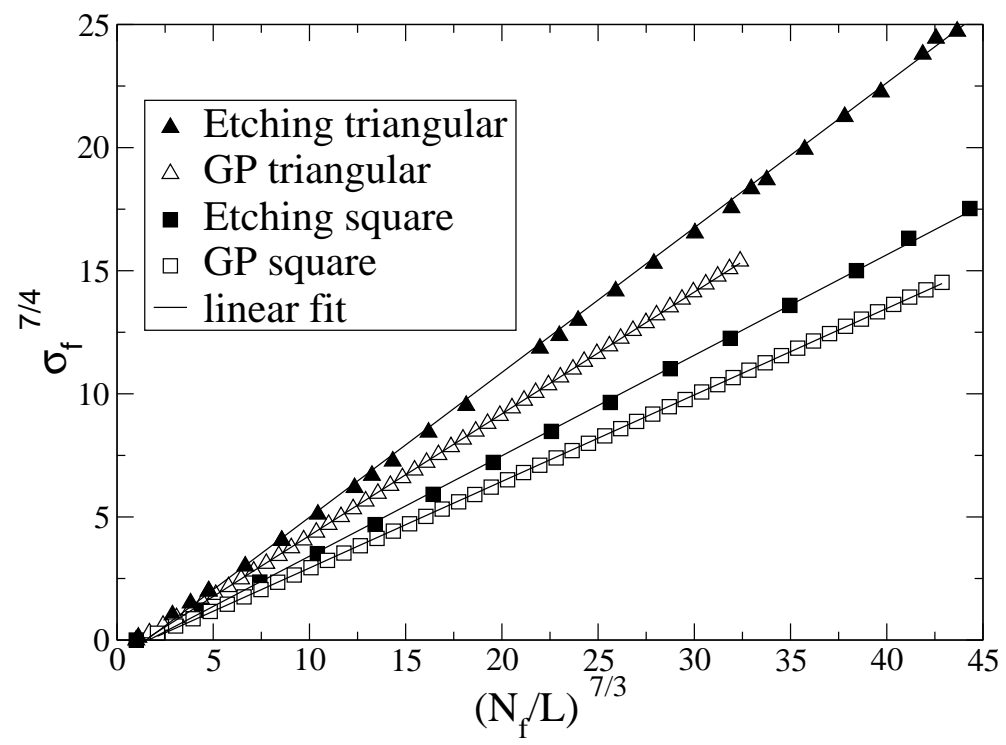

Figure 13. Extreme Gradients: GP (empty symbols) versus EGP (filled symbols) for square and triangular geometries.
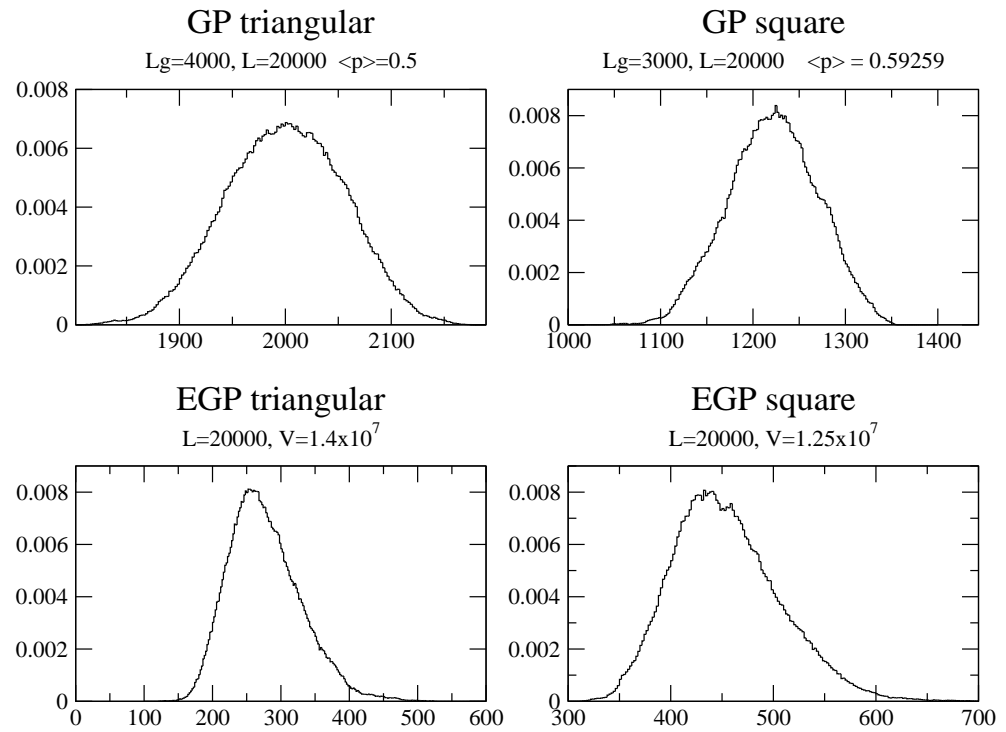

Figure 14. Depth distribution for the sites of the final front. Note the asymmetry for the EGP model, in contrast to the symmetric GP distributions.

\section{Discussion and Conclusion}

We have seen above that the detailed study of the extrapolation from the best numerical values corresponding to $L_{g}=4$ or $L_{g}=5$ was, for the square lattice, compatible with the exact values within numerical uncertainties. At this point 


\begin{tabular}{|c|c|c|}
\hline square lattice & $A$ & $B$ \\
\hline EGP & 0.40808 & -0.67458 \\
\hline GP & 0.35142 & -0.58746 \\
\hline triangular lattice & $A$ & $B$ \\
\hline EGP & 0.58804 & -0.89278 \\
\hline GP & 0.49459 & -0.70487 \\
\hline
\end{tabular}

TABLE 4. Comparison between GP, EGP. The table shows the results of the fit performed with the relation (3) on the data shown in Fig. 13

one could conclude that the numerical results are compatible with the existence of a single mathematical law for the width dependence, this law being valid from $L_{g}=1$ to infinity. Note that the quality of the random number generator could intervene in the purely numerical results and uncertainties, but when we tested the case $L_{g}=2$ or $L_{g}=3$ we did obtain numerically the exact results. Still, as long as a mathematical proof has not been given, it is not possible to conclude on the exact values of the coefficients $a$ and $b$.

The question of a unique mathematical power law can also be addressed from the exact results only. One can note that, remarkably, each time one tries to fit an observable $y$ (as $\left(N_{f} / L\right)^{7 / 3}$ or $\left.\sigma^{7 / 4}\right)$ using a function of $L_{g}$ like:

$$
y=a\left(L_{g}+b\right)
$$

the values of the term $b$ in the fit of the width are close to -1 (see Table 31). If the above law exists from $L_{g}=1$ to infinity, it suggests that the real value of $b$ is exactly -1 as the width is null in the trivial case $L_{g}=1$. As we have exact values, one can compute the equation of the line $y=a\left(L_{g}+b\right)$ defined by the two points $\left(2, \sigma_{f}(2)^{7 / 4}\right)$ and $\left(3, \sigma_{f}(3)^{7 / 4}\right)$. One obtains $a=0.294$ and $b=-1.055$. These values are close to the values obtained from the above numerical fit $(a=0.297$ and $b=-1.09$ ), and here again $b$ is close but not equal to -1 . Why is there a small mismatch with the simplest law? The answer to this question is two-fold.

(1) It is possible that the exact power law is not valid for $L_{g}=1$ or both for $L_{g}=1$ and 2 . These cases could be "anormal" as in these cases the perimeter and the accessible perimeter are the same (i.e. there is no Grossman-Aharony effect [15]). It is then possible that these two cases cannot be explained by the same mathematical law.

(2) The small discrepancy could be related to the fact that a slightly different definition of the interface, leading to very similar results for large values of $L_{g}$ (where universality enters), can give slightly different exact values for $L_{g}=1,2,3$. Our frontier definition considers only the occupied sites. It gives to these sites a privilege role whereas one should also consider the frontier of the empty cluster. In fact this is not new in percolation studies [8, 10] where it was shown that the barycenter between the frontier of the occupied cluster and the frontier of the empty cluster was a more natural object. It notably permitted better computations of the percolation threshold. We have studied the statistical width of the local barycenter which can also be computed exactly for $L_{g}=2$ or 3 . The results show the same behavior as described above i.e. a value of $b$ close but not equal to 
-1 . The question remains then open to define the nature of the geometrical object which would really display a $b$ value exactly equal to -1 . The answer to that question would certainly have interesting consequences for the SP problem itself.

In summary, it has been shown that the classical power laws of gradient percolation can be extended to extreme gradients with the same fractal exponents although the systems present no fractal geometry. Several comments can be drawn on these results.

First, from the purely mathematical point of view, our results suggest that there exists a conservation law which stipulates that the length of the correlated frontier is strictly proportional to the gradient length. This hypothesis remains to be proved rigorously.

Secondly, in a wider theoretical frame, the fact that the exponents $4 / 7$ and $3 / 7$ are valid down to the smallest $L_{g}$ values (or the steepest gradients) suggests that these exponents play the same type of role here that the exponent $1 / 2$ intervening in the fluctuations of the sum of independent identical random variables. In that last case the exponent applies to any number of random variables starting from 1 , 2 or 3 up to infinity. The exponents $4 / 7$ and $3 / 7$ may then play a more important role than critical exponents which only exist in the thermodynamic limit.

Third, the fact that the same exponent has been found for the square and the triangular lattice (and in two different models) marks a universal behavior that, in principle, is quite unexpected here. Up to now, the main physical argument to explain universality, i.e. the independence of exponents from the microscopic details of the model, was based on the long range of the correlations which appear in the proximity of a critical point. In the extreme gradient case, on the contrary, the (geometrical) correlations of the interface are so small that one cannot justify the observed universality.

Finally, our study suggests an intrinsic method to determine whether a given rough interface belongs to gradient percolation, without knowledge of the gradient and whatever the width of the interface. This can be very helpful to understand the properties of diffused contacts between materials.

\section{References}

[1] D. Stauffer and A. Aharony, "Introduction to Percolation Theory" 2nd ed.(Tailor and Francis, London, 1994) and references therein.

[2] B. Sapoval, M. Rosso and J.-F. Gouyet, J. Phys. Lett.(Paris), 46, L149 (1985). The gradient percolation situation has been sometimes called "statistically inhomogeneous random medium", bringing confusion with standard percolation which really describes a statistically inhomogeneous random media in contrast with a deterministic or non-random inhomogeneous medium, like a layered compound.

[3] B. Sapoval, M. Rosso and J.-F. Gouyet, in "The Fractal Approach to Heterogeneous Chemistry", edited by D. Avnir (John Wiley and Sons Ltd., New York,1989).

[4] H. Saleur and B. Duplantier, Phys. Rev. Lett. 58, 2325 (1987).

[5] S. Smirnov and W. Werner (2001), Critical exponents for 2d percolation, Math. Res. Lett. 8, 729-744. (math.PR/0109120)

[6] L. Balázs, Phys. Rev. E 54, 1183 (1996).

[7] B. Sapoval, S. B. Santra and Ph. Barboux, Europhys. Lett., 41, 297 (1998). S. B. Santra and B. Sapoval, Physica A., 266, 160-172 (1999).

[8] M. Rosso, J.-F. Gouyet and B. Sapoval, Phys. Rev. B 32, 6035 (1985).

[9] M. Rosso, J. Phys. A: Math. Gen., 22, L131 (1989)

[10] R. M. Ziff and B. Sapoval, J. Phys. A Math. Gen. 19, L1169-1172 (1986). 
[11] J. Quintanilla, S. Torquato and R. M. Ziff, J. Phys. A Math. Gen. 33, L399 (2000).

[12] J. Quintanilla, Phys. Rev. E 63, 061108 (2001).

[13] K. J. Falconer, "Fractal Geometry: Mathematical Foundations and Applications", Wiley, New York (1990).

[14] A. Gabrielli, A. Baldassarri, and B. Sapoval, Phys. Rev. E. 62, 3103 (2000).

[15] T. Grossman and A. Aharony, J. Phys. A 19, L745 (1986).

${ }^{1}$ Centre de Mathématiques et de leurs Applications, CNRS, Ecole Normale Supérieure, 94235 Cachan, France

$\dagger$ Agnes.Desolneux@Cmla.ens-CACHan.Fr

${ }^{2}$ Laboratoire de Physique de la Matière Condensée, CnRs, Ecole Polytechnique, 91128 Palaiseau, France

ฯ Bernard.Sapoval@polytechnique.Fr

3 infm, Unitá di Roma, Dipartimento di Fisica, Universitá "La Sapienza", P.le A.Moro 2, 00185 Roma, Italy

§ ANDREa.BAldASSARri@ROMA1.InFN.IT 